\title{
BORDISM INVARIANTS OF INTERSECTIONS OF SUBMANIFOLDS
}

\author{
BY \\ ALLAN HATCHER AND FRANK QUINN(1)
}

\begin{abstract}
This paper characterizes certain geometric intersection problems in terms of bordism obstructions. These obstructions give a setting in which to study such things as parametrized $h$-cobordisms (pseudoisotopy), and surgery above the middle dimension and on fibrations, where such intersection problems arise.
\end{abstract}

0. Introduction. Suppose $P$ and $Q$ are c.osed manifolds (smooth or PL) embedded or immersed in a manifold $M$. We give two techniques for changing the intersection of $P$ and $Q$ in $M$ by ambient isotopy or regular homotopy of $Q$. The first, generalizing lemmas of Stallings and Wall, characterizes the dimensions in which modifications of $P \cap Q$ (for example, making $P$ and $Q$ disjoint) by homotopy of $Q \hookrightarrow M$ are realized by ambient isotopy or regular homotopy of $Q \hookrightarrow M$. The second method, generalizing the classical Whitney procedure for cancelling pairs of isolated double points, characterizes the possible changes in $P \cap Q$ in terms of a bordism group for a metastable range of dimensions. As a particular case, when $P$ and $Q$ are sufficiently highly connected, the bordism group is the $k$-dimensional framed bordism group of the loop space of $M$, where $k=\operatorname{dim}(P \cap Q)$. For example, in the classical case $k=0$ this gives the integral group ring $\mathbf{Z}\left[\pi_{1} M\right]$. A similar but more complicated bordism invariant is obtained for the problem of modifying self-intersections of an immersion of $Q$ by regular homotopy.

In view of applications to parametrized versions of the $h$-cobordism theorem and surgery theory, we consider in the final section the situation when $P, Q$, and $M$ are fibered over some manifold, with all immersions, embeddings, etc., fiber preserving. We show in this case that the fibered theory is identical with the unfibered. As an immediate simple application we give some remarks on sections of metastable PL and vector bundles.

Since this paper was written, we have discovered that it overlaps two theses

Received by the editors November 16, 1972 and, in revised form, October 25, 1973. AMS (MOS) subject classifications (1970). Primary 57C35, 57D40.

Key words and phrases. Isotopy, regular homotopy, intersections.

(1) Partially supported by National Science Foundation grant GP7952X3. 
written at the University of Paris at Orsay in 1971. First, Theorem 1.1 is a special case, with a shorter proof, of a result in the third cycle thesis of A. Tineo [10]. Second, the techniques of $\S \S 2$ and 4 are similar to those used by J. P. Dax [11], who considers the problem of homotoping maps to embeddings, rather than regularly homotoping immersions to embeddings. His results extend those of Haefliger [1], while ours are oriented toward the immersion and isotopy problems considered in [6] and [7].

1. Homotopy of $Q$. We work throughout in either the smooth or PL category.

Suppose $f: P \rightarrow M, g: Q \rightarrow M$ are differentiable or PL maps which are transversal. Then we denote the transversal pullback $\{(p, q) \in P \times Q \mid f(p)=$ $g(q)\}$ together with its induced manifold structure by $f \mp g$.

1.1. Theorem. Suppose $i_{P}: P^{p} \rightarrow M^{m}$ and $i_{Q}: Q^{q} \rightarrow M^{m}$ are immersions (embeddings) of closed manifolds $P, Q$, and $M$. If $i_{Q}$ is homotopic to a map transversal to $i_{P}$ with pullback $N$, and $m>q+p / 2+1$, then $i_{Q}$ is regularly homotopic (ambient isotopic) to an immersion (embedding) transversal to $i_{P}$ with pullback $N$.

Proof. Let $H: Q \times I \rightarrow M$ be the homotopy specified in the theorem, so that $H_{0}=i_{Q}$ and $H_{1} \pitchfork i_{P}=N$. We may assume that the singular set

$$
\Sigma H=\{x \in Q \times I \mid H \text { is not an immersion (embedding) at } x\}
$$

is a subcomplex of dimension $2 q-m+1(2 q-m+2)$. (For the PL case see Stallings [6] or Hudson [4] on general position.) For $K \subset Q \times I$ define the shadow

$$
\operatorname{sh}(K)=\left\{(x, t) \in Q \times I \mid\left(x, t^{\prime}\right) \in K \text { for some } t^{\prime}<t\right\} .
$$

Case I. $\operatorname{dim} \operatorname{sh}(\Sigma H)<\operatorname{codim} P$, or $m>q+p / 2+1(m>q+p / 2+$ $3 / 2$ for embeddings). In this case we can approximate $H$ so that $\overline{\operatorname{sh}(\Sigma H)} \cap$ $H^{-1}(P)=\varnothing$. This is done by composing $H$ with a small ambient isotopy of $M$ which carries $\overline{H(\operatorname{sh}(\Sigma H))}$ off $P$.

Now since $\operatorname{sh}(\Sigma H)$ is "convex upwards", there is a function $\phi: Q \rightarrow I$ so that the closure of $\{(x, t) \mid t>\phi(x)\}$ is a neighborhood of $\overline{\operatorname{sh}(\Sigma H)}$ disjoint from $H^{-1}(P)$ and so that $\phi(x)=0$ if $(x, 0) \in \Sigma H$. Then $H^{\prime}: Q \times I \rightarrow M$, $H^{\prime}(x, t)=H(x, t \phi(x))$ is a regular homotopy (isotopy) of $i_{Q}$. Moreover $\left(H_{1}^{\prime}\right)^{-1}(P)=N$, since this is the only intersection of the graph of $\phi$ with $H^{-1}(P)$.

Case II. Embeddings, with $\operatorname{dim} \operatorname{sh}(\Sigma H) \leqslant \operatorname{codim} P$, or $m>q+p / 2+1$. If $\operatorname{codim} P=\operatorname{dim} \operatorname{sh}(\Sigma H)$ then in general position we will still have $\Sigma H \cap$ 
$H^{-1}(P)=\varnothing$, but there may be isolated intersections of $H^{-1}(P)$ with the shadows of top dimension cells of $\Sigma H$, which consist of immersed double points. If $p<m-1$, then in general position $H^{-1}(P)$ will intersect the shadow of at most one of each pair of double points-just move $H^{-1}(P)$ slightly near one double point of each pair-and, after excising from $\Sigma H$ small neighborhoods of these isolated double points whose shadows intersect $H^{-1}(P)$, we can proceed as before.

Finally, if $p \geqslant m-1$ and $m>q+p / 2+1$ then $m \geqslant 2(q+1)$, so $\Sigma H$ consists entirely of isolated double points. In general position no two of these double points will lie on the same level $Q \times\{s\}$, and $H$ itself will be the desired isotopy.

Previous versions of this theorem have been given by Stallings, when $P, Q$ are spheres and $p+q=m \geqslant 5$ [6, p. 246], Wall, generally when $p+q=m$ [8], and Laudenbach, when $N=\varnothing$ [5]. Our proof is essentially that of Stallings and Wall.

Theorem 1.1 implies that in the given dimension range if an embedding or immersion is homotopic to a map disjoint from a submanifold, then it is isotopic or regularly homotopic to a disjoint map. We next give a proposition showing this statement remains valid for immersions outside this range, and an example to show it generally fails for embeddings.

1.2. Proposition. Suppose $i_{Q}: Q^{q} \rightarrow M^{m}$ and $i_{P}: P^{p} \rightarrow M^{m}$ are immersions and $Q$ is closed. If $q \neq m-1$ and $i_{Q}$ is homotopic to a map disjoint from $P$, then it is regularly homotopic to an immersion disjoint from $P$.

Proof. Let $H$ be the homotopy, with $H_{0}=i_{Q}$ and $H_{1}(Q) \cap P=\varnothing$. The derivative of $i_{Q}$ gives an injective bundle map $d i_{Q}: \tau_{Q} \rightarrow \tau_{M}$ covering $i_{Q}$. Since homotopic bundles are isomorphic, we get a homotopy of bundle injections covering $H$. In particular, over $Q \times\{1\}$ we get $H_{1}: Q \rightarrow M-P$ covered by a bundle injection $b: \tau_{Q} \rightarrow \tau_{M-P}$. By the immersion classification theorem [2], [3] if $q \leqslant m-1, H_{1}$ is homotopic to an immersion $i_{Q}^{\prime}$ in $M-P$ with derivative homotopic to $b$. Considered as an immersion in $M$, however $i_{Q}^{\prime}$ is homotopic to $i_{Q}$ and has a covering homotopy of the derivative. By the classification theorem again the two immersions are regularly homotopic, provided $q \leqslant m-2$. If $q=m$ the result is trivial.

1.3. ExAmple. There are embeddings

$$
i_{1}: S^{k} \times S^{n} \times S^{j} \rightarrow S^{1} \times S^{2 k} \times S^{n} \times S^{j}
$$

and

$$
i_{2}: S^{2 k} \times S^{n} \rightarrow S^{1} \times S^{2 k} \times S^{n} \times S^{j}
$$

for all $n \geqslant 0, j \geqslant 0$, and $k \geqslant 1$, such that $i_{1}$ is homotopic to a map disjoint 
from $i_{2}$, but $i_{2}$ is not homotopic to a map disjoint from $i_{1}$.

This example, a generalization of one of Laudenbach [5], satisfies all but the dimension requirements of 1.1 with $i_{1}=i_{Q}, i_{2}=i_{P}$, and $N=\varnothing$. The conclusion of 1.1 for the case of embeddings and ambient isotopies fails, since the inverse of an ambient isotopy of $i_{1}$ off $i_{2}$ would give a homotopy of $i_{2}$ off $i_{1}$. In the notation of 1.1 we get an example for every $p$ and $q$ with $m \leqslant$ $q+p / 2+1, m-p \geqslant 1$, and $m-q \geqslant 2$.

Construction of 1.3. Let $i_{2}$ be the standard inclusion of a factor. For $i_{1}$ map $D^{k+1}$ in $S^{1} \times S^{2 k}$ by taking the disc around the $S^{1}$ factor to intersect itself in an arc. This gives a "self-linked" embedding of $\partial D^{k+1}=S^{k}$. Explicitly $\bar{i}_{1}$ is obtained as follows: $S^{1} \times S^{2 k} \supset S^{1} \times \mathrm{C}^{k}, \mathrm{C}^{k} \supset \mathrm{R}^{k} \supset D^{k}$, and $S^{1}$ is considered as $S^{1} \subseteq \mathrm{C}$. Now on $D^{k} \times I$ define $\overline{i_{1}}$ by

$$
\bar{i}_{1}(x, t)=\left(e^{3 \pi i t}\left\{\begin{array}{l}
x, \text { if } t \leqslant 1 / 3 \\
\left(e^{(3 t-1) \pi i / 2}\right) x, \quad 1 / 3 \leqslant t \leqslant 2 / 3 \\
i \cdot x, \text { if } t \geqslant 1 / 3
\end{array}\right) \in S^{1} \times \mathbf{C}^{k}\right.
$$

Now define $i_{1}=\overline{i_{1}} \times 1_{S^{n} \times s^{j}}$. By construction (using the disc $D^{k+1}$ ) $i_{1}$ is homotopic to projection on $S^{n} \times S^{j}$ composed with an inclusion as a factor. By including over a different point in $S^{1}$ from that used for $i_{2}$, this is clearly disjoint from $i_{1}$.

Next we show $i_{2}$ is not homotopically disjoint from $i_{1}$. The universal cover of $S^{1} \times S^{2 k}$ is $R \times S^{2 k}$, and the inverse image of the disc used to construct $i_{1}$ is a "chain" of discs. For $D^{2 k} \subset S^{2 k}, \mathbf{R} \times D^{2 k}=\mathrm{R} \times D^{k} \times D^{k}$, and these discs can be described by

$$
\bigcup_{p}\left[2 p-\frac{2}{3}, 2 p+\frac{2}{3}\right] \times D^{k} \times\{0\} \bigcup_{p}\left[2 p+1-\frac{2}{3}, 2 p+1+\frac{2}{3}\right] \times\{0\} \times D^{k}
$$

where $p \in \mathbf{Z}$. Now fold up by projecting to $[0,1] \times S^{2 k}$ by

$$
(t, v) \rightarrow\left(\left\{\begin{array}{l}
t-[t],[t] \text { even } \\
1-t+[t],[t] \text { odd }
\end{array}\right\}, v\right)
$$

This projects the boundary spheres of the chain of discs exactly to two linked discs $D_{1}^{k}, D_{2}^{k}$ with boundaries on opposite ends of $[0,1] \times S^{2 k}$. It is not hard to see that the inclusion $[0,1] \times S^{2 k}-\left(D_{1}^{k} \cup D_{2}^{k}\right) \rightarrow[0,1] \times S^{2 k}$ is homotopy equivalent to the standard map $S^{k} \times S^{k} \rightarrow S^{2 k}$ of degree one. Now a homotopy of $i_{2}$ disjoint from $i_{1}$ would, after lifting to the cover and folding, give a lift of the map $S^{2 k} \times S^{n} \rightarrow S^{2 k} \times S^{n} \times S^{j}$ to $S^{k} \times S^{k} \times S^{n} \times S^{j}$. In particular, it would give a right inverse for the map $S^{k} \times S^{k} \rightarrow S^{2 k}$. This is impossible because, among other things $\pi_{2 k}\left(S^{2 k}\right)$ is infinite, while $\pi_{2 k}\left(S^{k} \times S^{k}\right)=$ $2 \pi_{2 k}\left(S^{k}\right)$ is always finite. 
2. Bordism of $P \cap Q$. Let $f: P \rightarrow M, g: Q \rightarrow M$. be maps of topological spaces. The homotopy pullback is $E(f, g)=\{(p, q, \theta) \mid p \in P, q \in Q$, and $\theta:[0,1]$ $\rightarrow M$ with $\theta(0)=f(p), \theta(1)=g(q)\}$. This gives a homotopy commutative diagram

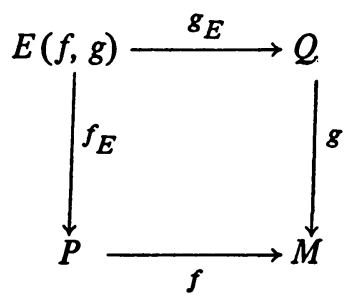

which is universal in the sense that if $h_{1}: X \rightarrow P, h_{2}: X \rightarrow Q$ are maps, and a homotopy $f h_{1} \sim g h_{2}$ is given, then there is a natural map $j: X \rightarrow E(f, g)$ so that $h_{1}=f_{E} j, h_{2}=g_{E} j$, and the homotopy from $f h_{1}$ to $g h_{2}$ is $j$ composed with the homotopy from $f f_{E}$ to $g g_{E}$. For example, if $f, g$ are transversal maps of manifolds, then there is a natural map $f$ $\mathrm{T} g \rightarrow E(f, g)$.

Next we define "framed bordism with coefficients in a bundle". Let $X$ be a space with a (PL or vector) bundle $\xi$ over it. Define $\Omega_{*}^{f r}(X ; \xi)$ to be the bordism groups of manifolds mapping to $X$, together with a stable bundle isomorphism of the normal bundle with the pullback of $\xi$. This is natural with respect to maps covered by stable bundle maps. The example which will arise is $\Omega_{*}^{f r}\left(E(f, g) ; v_{P} \oplus\right.$ $\left.\nu_{Q} \oplus \tau_{M}\right)$. The indicated bundle is a shorthand for $f_{E}^{*} \nu_{P} \oplus g_{E}^{*} \nu_{Q} \oplus f_{E}^{*} f^{*} \tau_{M}$ ( $\nu$ and $\tau$ are normal and tangent bundles respectively). Generally to simplify formulas we will omit notation indicating pullback of bundles.

If $\xi$ is a $k$-dimensional bundle over $X$, and $e$ the trivial bundle, then $\Omega_{*}^{f r}(X ; \xi) \simeq \lim \pi_{*+j} T\left(\xi \oplus e^{j-k}\right)$. This is a version of the usual Pontrjagin-Thom theorem ( $T$ denotes Thom spaces). Thus the group depends only on the fiber homotopy type of $\xi$.

2.1. PRoposition. If $f: P^{p} \rightarrow M^{m}$ and $g: Q^{q} \rightarrow M^{m}$ are transversal maps of closed manifolds, then the transversal pullback $f \mp g$ determines a bordism class $[f \bar{\pi} g]$ in $\Omega_{p+q-m}^{f r}\left(E(f, g) ; \nu_{P} \oplus \nu_{Q} \oplus \tau_{M}\right)$ which is an invariant of the homotopy classes of $f$ and $g$.

P ROOF. The universal property of $E(f, g)$ gives a map of $f \bar{\pi} g$ to $E(f, g)$. Further, the transversal pullback of homotopies of $f$ or $g$ will also map to $E(f, g)$ giving a bordism between the pullbacks of homotopic maps.

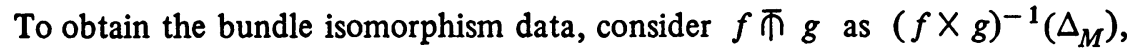
the pullback of the diagonal $\Delta_{M}$ under $f \times g: P \times Q \rightarrow M \times M$. Then there is a natural splitting

$$
v_{f} \pi_{g} \simeq v_{P \times Q} \oplus \nu(f \pi g, P \times Q) \simeq v_{P} \oplus v_{Q} \oplus \tau_{M} .
$$


If $f$ and $g$ are immersions, the bundles $\nu_{P} \oplus \nu_{Q} \oplus \tau_{M}$ and $\nu(P, M) \oplus$ $\nu(Q, M) \oplus \nu_{M}$ are stably isomorphic over $E(f, g)$. We will use the second bundle from now on, since it arises more directly from the geometry of the situation.

2.2. Theorem. If $i_{P}: P^{p} \rightarrow M^{m}$ and $i_{Q}: Q^{q} \rightarrow M^{m}$ are transversal immersions (embeddings) of closed manifolds in $M, m>p+q / 2+1, m>$ $q+p / 2+1$, and $N$ is equivalent to $i_{P} \bar{\pi} i_{Q}$ in

$$
\Omega_{p+q-m}^{f r}\left(E\left(i_{P}, i_{Q}\right) ; \nu(P, M) \oplus \nu(Q, M) \oplus \nu_{M}\right),
$$

then there is a regular homotopy (ambient isotopy) of $i_{Q}$ to an immersion (embedding) $i_{Q}^{\prime}$ transversal to $i_{P}$ with $i_{P}$ 币 $i_{Q}^{\prime}$ diffeomorphic to $N$.

Proof of 2.2. (i) To begin, we put $i_{Q}$ in general position with respect to $i_{P}$ so that $i_{P} \bar{\pi} i_{Q}$ is embedded in $M$ by $i_{P}$ and $i_{Q} ; i_{P} \bar{\pi} i_{Q}$ can then be identified with $i_{P}(P) \cap i_{Q}(Q)$. This is accomplished by making $P$ and $Q$ disjoint from the self-intersections of the other in $M$. For this general position suffices if $\operatorname{dim}($ self-intersections of $P$ ) $<\operatorname{codim} Q$ or $2 p-m<m-q$, and $\operatorname{dim}($ self-intersections of $Q$ ) $<\operatorname{codim} P$ or $2 q-m<m-p$.

Next, let $W$ be a bordism realizing the equivalence of $i_{P} \Phi i_{Q}$ and $N$ in $\Omega_{p+q-m}^{f r}\left(E\left(i_{p}, i_{Q}\right) ; \nu(P, M) \oplus \nu(Q, M) \oplus \nu_{M}\right)$. Thus we have a map $H$ : $W \times I \rightarrow M$ restricting to $H_{0}: W \times\{0\} \rightarrow i_{P}(P)$ and $H_{1}: W \times\{1\} \rightarrow i_{Q}(Q)$ with $H \mid\left(i_{P}\right.$ 雨 $\left.i_{Q}\right) \times I$ the constant homotopy.

(ii) Approximate $H_{0}$ by an embedding in $i_{P}(P)$ extending the inclusion $i_{P} \bar{\pi} i_{Q} \hookrightarrow M$ and disjoint from the self-intersections of $P$. This is possible if $\operatorname{dim} W<p / 2, \operatorname{dim} W+\operatorname{dim}\left(\right.$ self-intersections of $P$ ) $<p$. Similarly, make $H_{1}$ an embedding.

(iii) Approximate $H$ by an embedding (with $\left(i_{P} \mp i_{Q}\right) \times I$ pinched to $i_{P}$ 币 $\left.i_{Q} \hookrightarrow M\right)$ extending $H_{0}$ and $H_{1}$ and intersecting $i_{P}(P)$ and $i_{Q}(Q)$ only at $H_{0}$ and $H_{1}$. This uses $\operatorname{dim}(W \times I)<m / 2$ and $\operatorname{dim}(W \times I)<$ $\operatorname{codim} P, \operatorname{codim} Q$.

(iv) Split $v(W \times I, M)$ as $\nu(W, Q) \oplus v(W, P) \times I$ compatibly with the natural splitting $v\left(i_{P} \pi i_{Q}, M\right) \approx v\left(i_{P} \pi i_{Q}, Q\right) \oplus v\left(i_{P} \pi i_{Q}, P\right)$. This is done as follows. By hypothesis

$$
v_{W} \approx \nu(W, M) \oplus v_{M} \approx \nu(P, M) \oplus \nu(Q, M) \oplus v_{M},
$$

so $\nu(W, M) \oplus e \approx \nu(P, M) \oplus \nu(Q, M)$. Since $\nu(W, M) \oplus e \approx \nu(P, M) \oplus$ $\nu(W, P) \oplus e$ also, $\nu(Q, M) \approx \nu(W, P) \oplus e$. Now

$$
\nu(W \times I, M) \oplus e \oplus e \approx \nu(W, M) \oplus e \approx \nu(W, Q) \oplus \nu(Q, M) \oplus e
$$

which equals $\nu(W, Q) \oplus \nu(W, P) \oplus e \oplus e$. Destabilizing, $\nu(W \times I, M) \approx$ 
$\nu(W, Q) \oplus \nu(W, P)$ which,- taking into account our convention of omitting notation for pullbacks of bundles, is really $\nu(W, Q) \oplus \nu(W, P) \times I$. Note that destabilizing is well defined since $\nu(W, Q)$ and $\nu(W, P)$ are stable bundles $(\operatorname{dim} W<\operatorname{dim} \nu(W, Q), \operatorname{dim} \nu(W, P))$.

Steps (i)-(iv) allow us to build a simple model for the desired deformation of $i_{Q}$. First extend the embedding $W \times I \hookrightarrow M$ to include a small collar neighborhood to obtain $W^{+} \times I^{+} \hookrightarrow M$. Let $(x, y, t) \in \nu\left(W^{+}, Q\right) \oplus$ $v\left(W^{+}, P\right) \times I$ be coordinates for $v\left(W^{+} \times I^{+}, M\right)$, so that $W^{+} \times I^{+}$has coordinates $(0,0, t), Q$ has coordinates $(x, 0,0)$, and $P$ has coordinates $(0, y, \phi)$ for some function $\phi: W^{+} \rightarrow I^{+}$with $\phi^{-1}(0)=i_{P} \bar{\pi} i_{Q}$ and $\phi^{-1}(1)=N$. Let $\psi: v\left(W^{+}, Q\right) \rightarrow I$ equal zero away from $W$ and one near $W$. Then $(x, 0,0) \mapsto(x, 0, s \psi(x)), 0 \leqslant s \leqslant 1$, provides an isotopy of $i_{Q}$ near $W$ which replaces $i_{P}$ 而 $i_{Q}$ by $N$.

REMARKS. (1) The regular homotopy (isotopy) constructed not only ends with an immersion (embedding) having the desired intersection $N$, but the intersection $W$ of the regular homotopy (isotopy) itself can be preassigned. Thus for example, any element of the bordism group can be realized as the intersection of a regular homotopy (isotopy) between two disjoint immersions (embeddings).

(2) Example 1.3 gives counterexamples to the statement of 2.2 for embeddings outside the given dimension range.

(3) If $P, Q, M$ have boundary, then 2.2 can be modified to hold a neighborhood of the boundary fixed (the invariant is defined via a difference construction, see 4.2), or to allow part of the boundary to vary using a relative bordism group.

A little elaboration is required to treat self-intersections. Suppose $i: Q \rightarrow$ $M$ is an immersion which is self-transversal in the sense that $i \times i:\left(Q \times Q-\Delta_{Q}\right)$ $\rightarrow M \times M$ is transversal to the diagonal $\Delta_{M}$. In this case define the self-intersection $i$ 开 $i$ to be the manifold $\left\{\left(q_{1}, q_{2}\right) \mid q_{1} \neq q_{2}\right.$ and $\left.i\left(q_{1}\right)=i\left(q_{2}\right)\right\} / Z / 2$. Here $/ Z / 2$ means divide out by the free $Z / 2$ action interchanging $\left(q_{1}, q_{2}\right)$ and $\left(q_{2}, q_{1}\right)$. The self-inverse image $(i \bar{\pi} i)^{\wedge}$ is $(i \times i)^{-1}\left(\Delta_{M}\right)-\Delta_{Q}$ and double covers $i$ 雨 $i$. If $i$ has no triple points, then $i$ i $i$ is a submanifold of $M$, while $(i \bar{\pi} i)^{\wedge}$ is a submanifold of $Q$.

The homotopy pullback

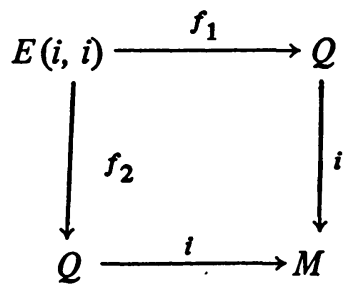

also has a $Z / 2$ action, not free, by interchanging the copies of $Q$. Explicitly 
this is $I=(p, q, \theta)=\left(q, p, \theta^{-1}\right)$ where $\theta^{-1}$ denotes the path with reversed parametrization $\left(\theta^{-1}(t)=\theta(1-t)\right)$. The natural map $(i \bar{\Pi} i)^{\wedge} \rightarrow E(i, i)$ is equivariant. The freeness of the action on $(i \bar{\Gamma} i)^{\wedge}$ is captured as follows: Let $W_{2}$ be a free acyclic $\mathrm{Z} / 2$ complex (e.g., $W_{2}=S^{\infty}$ with the antipodal map, so $\left.W_{2} / \mathrm{Z} / 2=\mathbf{R} P^{\infty}\right)^{-}$Let $E(i, i) \mathrm{X}_{2} W_{2}$ denote the quotient of the product by the diagonal $\mathrm{Z} / 2$ action. The map $E(i, i) \mathrm{X}_{2} W_{2}$ is universal in the following sense: given a map $g: X \rightarrow M$, a double cover $\pi: \hat{X} \rightarrow X$, a map $h: \hat{X} \rightarrow Q$, and a homotopy from $i \circ h$ to $g \circ \pi$, then there is a canonical map $f: X \rightarrow$ $E(i, i) \mathrm{X}_{2} W_{2}$ such that $\hat{X}$ is induced from the double cover of $E(i, i) \times_{2} W_{2}$, and the maps $g, h$, and the homotopy from $i \circ h$ to $g \circ \pi$ are all given by composing $j$ with the corresponding maps and homotopy defined on $E(i, i) \times_{2} W_{2}$.

The natural map $j: i$ 币 $i \rightarrow E(i, i) \mathrm{X}_{2} W_{2}$ will be our characteristic bordism element once some bundle information is included.

Denote the normal bundle $\nu(Q, M)$ by $\nu$, then as before the stable normal bundle of $(i \bar{T} i)^{\wedge}$ is naturally isomorphic with the pullback of $f_{1}^{*} \nu \oplus$ $f_{2}^{*} \nu \oplus f^{*} \nu_{M}$ from $E(i, i)$. The involution on $E(i, i)$ is covered by the bundle involution $I^{*}$ which interchanges the two factors $f_{1}^{*} \nu$ and $f_{2}^{*} \nu$, and an involution $\omega$ of $f^{*} \nu_{M}$.

Taking the quotient, we have constructed a stable bundle map

$$
\nu_{i \pi_{i}} \rightarrow\left[f_{1}^{*} \nu \oplus f_{2}^{*} \nu\right] / I^{*} \oplus f^{*} \nu_{M} / \omega \cdot(2)
$$

2.3 THEOREM. If $i: Q^{q} \rightarrow M^{m}$ is an immersion of a closed manifold with $m>3 q / 2+1$ and $N$ is equivalent to $i \bar{\pi} i$ in

$$
\Omega_{2 q-m}^{f r}\left(E(i, i) X_{2} W_{2} ;\left(\left[f_{1}^{*} \nu \oplus f_{2}^{*} \nu\right] / I^{*}\right) \oplus\left(f^{*} v_{M} / \omega\right)\right),
$$

then $i$ is regularly homotopic to an immersion with self-intersection $N$.

Proof. Let $W$ be a bordism of the indicated sort between $i \pi i$ and $N$, and let $\hat{W}$ be its double cover. Suppose first that this is a trivial cover: $\hat{W}=$ $W_{+} \cup W_{-}$and $\mathbf{Z} / 2$ interchanges the two pieces. Approximate $\hat{W} \rightarrow Q$ by an embedding (keeping $i$ T $i$ fixed), then 2.2 applies to a neighborhood $Q_{+}$of $W_{+}$in $Q$ to isotope it to have intersection $N$ with $Q-Q_{+}$. This gives an immersion with self-intersection $N$.

In the general case when $\hat{W} \rightarrow W$ is not a trivial cover, it is still locally trivial and the theorem will follow by applying the above considerations locally. Put a handlebody structure on $(W, i \bar{T} i)$; then the inverse image in $\left(\hat{W},(i \bar{\pi} i)^{\wedge}\right)$ is a handlebody structure in which disjoint handles are interchanged by the $Z / 2$

(2)We would like to thank the referee for correcting an error in our description of this bundle. 
action. Now we can embed a pair of the lowest dimensional handles disjointly, and use the discussion above to obtain a regular homotopy in a neighborhood of one handle disjoint from the other which moves across the handle. This gives a new situation $\left(W^{\prime}, i^{\prime} \overline{i^{\prime}}\right)$ with one fewer handle. Induction on the number of handles completes the proof.

REMARK. The bordism class of $i \pi i$ in $\Omega_{2 q-m}^{f r}\left(E(i, i) X_{2} W_{2}\right.$; $\left.\left(\left[f_{1}^{*} \nu \oplus f_{2}^{*} \nu\right] / I^{*}\right) \oplus\left(f^{*} \nu_{M} / \omega\right)\right)$ is not an invariant of the homotopy class of $i$, but only of the regular homotopy class. For example, a self-transverse immersion $i: S^{n} \rightarrow S^{2 n}$ with $i$ 币 $i$ consisting of one point is homotopic to an embedding but not regularly homotopic to one. (The bordism group in this case is $\mathbf{Z}$ or $\mathbf{Z} / 2$, depending on the parity of $n$.) This example also shows that the analogue of 1.1 for self-intersections of immersions is false.

To get a homotopy invariant we would have to allow maps $Q \rightarrow M$ having singularities. This is the situation studied by Haefliger [1]. Haefliger's theorem presumably would fit into this context by using a relative bordism group to allow for the singularities.

3. Highly connected submanifolds. In this section we elaborate on the bordism invariants of the preceding section when $P$ and $Q$ are highly connected. In particular, the bordism groups will be seen to depend only on $M$, and not on $P, Q$, or the immersions $i_{P}, i_{Q}$. In this case the obstructions for different immersions can be compared, and formulas similar to those of $[7, \S 5]$ are derived.

If $M$ is also highly connected, the bordism obstruction group collapses to $\Omega_{p+q-m}^{f r}(*)$. In this case our results generalize a theorem of Wells [9].

Let $*_{P} \in P,{ }_{Q} \in Q$, and $* \in M$ be basepoints and choose paths from $i_{P}\left(*_{P}\right)$ and $i_{Q}\left(*_{Q}\right)$ to $*$. The basepoints induce a map $E\left(*_{P},{ }_{Q}\right) \rightarrow$ $E\left(i_{P}, i_{Q}\right)$, and the paths give a homotopy equivalence

$$
E\left(*_{P}, *_{Q}\right)=C\left(I, 0,1 ; M, i_{P}\left(*_{P}\right), i_{Q}\left(*_{Q}\right)\right) \stackrel{\cong}{\rightarrow} \Lambda(M, *) .
$$

(Here $\Lambda(M, *)$ denotes the loopspace of $M$ at *) Choose orientations of $P$ at ${ }_{P}^{*}, Q$ at ${ }^{*}$, and $M$ at $*$. Via the paths from $i_{P}\left(*_{P}\right)$ and $i_{Q}\left({ }_{Q}\right)$ to $*$, these induce framings of $\nu(P, M)$ at $i_{P}\left({ }^{*}\right)$ and $\nu(Q, M)$ at $i_{Q}\left({ }^{*}\right)$. The orientation of $M$ at $*$ gives a framing of $\nu_{M}$ at $*$ which pulls back along the paths to framings of $v_{M}$ at $i_{P}\left(*_{P}\right)$ and $i_{Q}\left(*_{Q}\right)$. Choosing the framing of $\nu_{M}$ at $i_{Q}\left({ }^{*}\right)$, there is then induced a framing of $\nu(P, M) \oplus$ $\nu(Q, M) \oplus \nu_{M}$ over $E\left(*_{P},{ }_{Q}\right) \simeq \Lambda(M, *)$. Thus we have a map

$$
\Omega_{*}^{f r}(\Lambda(M, *)) \rightarrow \Omega_{*}^{f r}\left(E\left(i_{P}, i_{Q}\right) ; \nu(P, M) \oplus \nu(Q, M) \oplus v_{M}\right) .
$$


This map is $k$-connected if $P$ and $Q$ are $(k+1)$-connected. So having made the choices above we may identify

$$
\left[i_{P} \pi i_{Q}\right] \in \Omega_{p+q-m}^{f r}\left(E\left(i_{P}, i_{Q}\right) ; \nu(P, M) \oplus \nu(Q, M) \oplus v_{M}\right)
$$

with an element $\lambda\left(i_{P}, i_{Q}\right) \in \Omega_{p+q-m}^{f r}(\Lambda(M, *))$ provided $P$ and $Q$ are $(p+q-m+1)$-connected. We shall next discuss the dependence of $\lambda\left(i_{P}, i_{Q}\right)$ on the choices.

There is a natural two-sided action of $\pi_{1}(M, *)$ on $\Omega_{*}^{f r}(\Lambda(M, *))$ via composition of loops on the left or on the right. Rechoosing the path from $i_{P}\left(*_{P}\right)$ to $*$ by $\sigma \in \pi_{1}(M, *)$ changes $\lambda\left(i_{P}, i_{Q}\right)$ by left multiplying by $\omega_{1}(\sigma) \sigma$. The sign $\omega_{1}(\sigma)$, which is +1 or -1 according to whether $\sigma$ preserves or reverses orientation in $M$, measures the change in the orientation of $\nu(P, M)$. Rechoosing the path from $i_{Q}\left({ }^{*}{ }_{Q}\right)$ to : by $\sigma$ changes $\lambda\left(i_{P}, i_{Q}\right)$ by right multiplying by $\sigma^{-1}$. There is no sign change here since orientation changes of $\nu(Q, M)$ and $\nu_{M}$ given by $\omega_{1}(\sigma)$ cancel.

Now we investigate the effect of interchanging $P$ and $Q$. Let $\omega$ : $\Lambda(M, *) \rightarrow\{0$ or PL $\}$ be the loop of the classifying map for $\nu_{M}$. Note that $\omega_{1}$ above is just $\pi_{0}(\omega): \pi_{0}(\Lambda(M, *)) \rightarrow \pi_{0}\{0$ or PL $\}$. Let $I: \Lambda(M, *) \rightarrow$ $\Lambda(M, *)$ denote loop inverse. From these we define an involution on $\Omega_{*}^{f r}(\Lambda(M, *)), \lambda \rightarrow \bar{\lambda}$, as follows: If $\lambda$ is represented by $f: X \rightarrow \Lambda(M, *)$, with $b$ a framing of $X$, then $\bar{\lambda}$ is represented by $I \circ f: X \rightarrow \Lambda(M, *)$ with $X$ framed by $(\omega f) \cdot b$, i.e., with the framing $b$ changed by $\omega f: X \rightarrow$ $\left\{0\right.$ or PL\}. This is indeed an involution since $\overline{\bar{\lambda}}$ is represented by $I^{2} f=$ $f: X \rightarrow \Lambda(M, *)$ framed by $(\omega I f)(\omega f) \cdot b=(\omega f)^{-1}(\omega f) \cdot b=b$.

In terms of this involution we have

$$
\lambda\left(i_{Q}, i_{P}\right)=(-1)^{(m-p)(m-q)} \overline{\lambda\left(i_{P}, i_{Q}\right)}
$$

The sign comes from permuting $\nu(P, M)$ and $\nu(Q, M)$, the loop inverse on $\Lambda(M, *) \simeq E\left(*_{P},{ }^{*} Q\right)$ is induced by interchange of $P$ and $Q$, and the reframing by $\omega$ is the result of pulling back the framing of $\nu_{M}$ over $E\left(*_{P},{ }_{Q}{ }_{Q}\right)$ from $i_{P}\left(*_{P}\right)$ rather than $i_{Q}\left({ }_{Q}\right)$.

Change of one of the choices of orientation at ${ }^{*},{ }^{*}{ }_{Q}$, or $*$ just changes the sign of $\lambda\left(i_{P}, i_{Q}\right)$.

For the self-intersection obstruction the corresponding bordism group is

$$
\Omega_{*}^{f r}\left(\Lambda(M, *) \mathrm{X}_{2} W_{2} ;\left[f_{1}^{*} \nu \oplus f_{2}^{*} \nu\right] / I^{*} \oplus f^{*} \nu_{M} / \omega,\right.
$$

where $\nu=\nu(Q, M)$ and the $Z / 2$ action on the loop space is loop inverse as above. Now however $\nu$ is framed, so $f_{1}^{*} \nu, f_{2}^{*} \nu$ are isomorphic. If we reparameterize by $\left(v_{1} v_{2}\right) \rightarrow\left(v_{1}+v_{2}, v_{1}-v_{2}\right)$, the involution $I^{*}$ becomes 
trivial on the first factor, multiplication by -1 on the second. Stably, then $\left[f_{1}^{*} \nu \oplus f_{2}^{*} \nu\right] / I^{*}$ is isomorphic to $m-q$ copies of the canonical line bundle $\gamma$ over $\mathbf{R} P^{\infty}$. On the twofold cover $\Lambda(M, *) \times W_{2} \simeq \Lambda(M, *)$ of $\Lambda(M, *) \mathrm{X}_{2} W_{2}, \nu_{M}$ has a framing, and the involution given by $I$ and $\omega$ covers the involution $I$. Thus $f^{*} v_{M} / \omega \simeq e / \omega$ on $\Lambda(M, *) \times W_{2}$. The selfintersection group is therefore

$$
\Omega_{*}^{f r}\left(\Lambda(M, *) \mathrm{X}_{2} W_{2} ; \gamma^{m-q} \oplus e / \omega\right)
$$

We sum all this up as a proposition.

3.1. Proposition. If $P$ and $Q$ are $(k+1)$-connected then a choice of basepoints, orientations, and connecting paths as above gives isomorphisms

$$
\begin{gathered}
\Omega_{k}^{f r}\left(E\left(i_{P}, i_{Q}\right) ; \nu(P, M) \oplus \nu(Q, M) \oplus \nu_{M}\right) \approx \Omega_{k}^{f r}(\Lambda(M, *)), \\
\Omega_{k}^{f r}\left(E\left(i_{Q}, i_{Q}\right) X_{2} W_{2} ;\left[f_{1}^{*} \nu \oplus f_{2}^{*} \nu\right] / I^{*} \oplus f^{*} \nu_{M} / \omega\right. \\
\approx \Omega_{k}^{f r}\left(\Lambda(M, *) \times_{2} W_{2} ; \gamma^{m-q} \oplus e / \omega\right) .
\end{gathered}
$$

Denote the image of $\left[i_{P} \bar{\pi} i_{Q}\right]$ by $\lambda\left(i_{P}, i_{Q}\right)$ if $k \geqslant p+q-m$, and $\left[i_{Q} \bar{\pi} i_{Q}\right]$ by $\mu\left(i_{Q}\right)$ if $k \geqslant 2 q-m$. Then if the paths from $i_{P}\left(*_{P}\right)$ or $i_{Q}\left({ }^{*}\right)$ are changed by a loop $\sigma, \lambda\left(i_{P}, i_{Q}\right)$ becomes $\omega_{1}(\sigma) \sigma \lambda\left(i_{P}, i_{Q}\right)$ or $\lambda\left(i_{P}, i_{Q}\right) \sigma^{-1}$ and $\mu\left(i_{Q}\right)$ becomes $\omega_{1}(\sigma) \sigma \mu\left(i_{Q}\right) \sigma^{-1}$. Finally

$$
\lambda\left(i_{Q}, i_{P}\right)=(-1)^{(m-p)(m-q)} \overline{\lambda\left(i_{P}, i_{Q}\right)} .
$$

We next give formulas relating the invariants $\lambda$ and $\mu$. First some definitions:

3.2. Definition. If $\zeta^{k}$ is a (block, PL, or vector) bundle over a closed manifold $Q^{q}$, then the "Euler class"

$$
\chi(\zeta) \in \Omega_{q-k}^{f r}\left(Q ; \zeta \oplus v_{Q}\right)
$$

is the bordism class of the intersection of two copies of the zero section of $\zeta$.

Here we have used the canonical identification $E\left(1_{Q} \cdot 1_{Q}\right) \simeq Q$.

Next let the homomorphisms

$$
\begin{gathered}
c_{k}: \Omega_{*}^{f r}\left(\Lambda(M, *) X_{2} W_{2} ; \gamma^{k} \oplus e / \omega\right) \rightarrow \Omega_{*}^{f r}(\Lambda(M, *)), \\
d_{k}: \Omega_{*}^{f r}(\Lambda(M, *)) \rightarrow \Omega_{*}^{f r}\left(\Lambda(M, *) X_{2} W_{2} ; \gamma^{k} \oplus e / \omega\right)
\end{gathered}
$$

be induced by the natural double cover and inclusion respectively. Finally, if $i_{Q_{1}}$ and $i_{Q_{2}}$ are immersions with chosen paths to $* \in M$, let $i_{Q_{1} \# Q_{2}}$ be the immersion obtained by connected sum along the chosen paths.

3.3. PROPOSITION. For immersions of $(2 q-m+1)$-connected q-manifolds, the invariants $\lambda$ and $\mu$ satisfy: 


$$
\begin{gathered}
\lambda\left(i_{Q_{1}}, i_{Q_{2} \# Q_{3}}\right)=\lambda\left(i_{Q_{1}}, i_{Q_{2}}\right)+\lambda\left(i_{Q_{1}}, i_{Q_{3}}\right), \\
\lambda\left(i_{Q_{2}}, i_{Q_{1}}\right)=(-1)^{m-q} \overline{\lambda\left(i_{Q_{1}}, i_{Q_{2}}\right),} \\
\mu\left(i_{Q_{1}} \# Q_{2}\right)=\mu\left(i_{Q_{1}}\right)+\mu\left(i_{Q_{2}}\right)+d_{m-q}\left(\lambda\left(i_{Q_{1}}, i_{Q_{2}}\right)\right), \\
\lambda\left(i_{Q}, i_{Q}\right)=c_{m-q}\left(\mu\left(i_{Q}\right)\right)+\chi(\nu(Q, M)),
\end{gathered}
$$

where

$$
\chi(\nu(Q, M)) \in \Omega_{2 q-m}^{f r}\left(Q ; \nu(Q, M) \oplus \nu_{M}\right) \approx \Omega_{2 q-m}^{f r}(*) \subset \Omega_{2 q-m}^{f r}(\Lambda(M, *)) .
$$

The homomorphisms $c_{k}$ and $d_{k}$ are related in the following ways:

3.4. Proposition. For each $k$

(1) $c_{k} d_{k}(a)=a+(-1)^{k} \bar{a}$ for $a \in \Omega_{*}^{f r}(\Lambda(M, *))$,

(2) there is a long exact sequence

$$
\begin{gathered}
\Omega_{*}^{f r}\left(\Lambda(M, *) X_{2} W_{2} ; \gamma^{k+1} \oplus e / \omega\right) \stackrel{c_{k+1}}{\longrightarrow} \Omega_{*}^{f r}(\Lambda(M, *)) \\
\Omega_{*}^{f r}\left(\Lambda(M, *) \times_{2} W_{2}: \gamma^{k} \oplus e / \omega\right)
\end{gathered}
$$

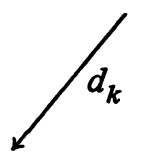

with $\partial$ of (graded) degree -1 .

Proof. We first define the map $\partial$ using the representation $W_{2}=S^{\infty}=$ $\bigcup_{k} S^{k}$. If $f: X \rightarrow \Lambda(M, *) \times_{2} W_{2}, b: \nu_{X} \rightarrow \gamma^{k} \oplus e / \omega$ represents an element of the self-intersection group, then the image of $f$ lies in some $\Lambda(M, *) \times_{2} S^{l}$. Contained in $\Lambda(M, *) \times_{2} S^{l}$ is $\Lambda(M, *) \times_{2} S^{l-1}$ as a subspace with neighborhood the total space of the line bundle $\gamma$. Then $\partial[X, f, b]$ is defined to be the transversal pullback of this subspace. Note that the number of copies of $\boldsymbol{\gamma}$ in the normal bundle increases by one.

The proof of exactness is straightforward. For example, if $d_{k}(a)=0$, then there is a bordism of $d_{k}$ of a representative of $a$ to zero. Essentially applying the $\partial$ construction to the bordism gives an element $b$ with $c_{k+1}(b)=a$.

We can apply 2.2 and 2.3 to give an embedding criterion for a family of immersions. Suppose $i_{1}, \cdots, i_{n}$ are immersions of closed $q$-manifolds $Q_{1}, \cdots, Q_{n}$ in $M$, with basepoints, orientations, and paths chosen as above. 
3.5. THEOREM. If $m>3 q / 2+1$ and each $Q_{j}$ is $(2 q-m+1)$-connected then $\left\{i_{j}\right\}$ is regularly homotopic to a family of disjoint embeddings if and only if

(1) $\lambda\left(i_{j}, i_{k}\right)=0$ in $\Omega_{2 q-m}^{f r}(\Lambda(M, *))$ for each $j \neq k$,

(2) $\mu\left(i_{j}\right)=0$ in $\Omega_{2 q-m}^{f r}\left(\Lambda(M, *) X_{2} W_{2} ; \gamma^{m-q} \oplus e / \omega\right)$ for each $j$.

Note that (1) and (2) are independent of the choices of orientations, etc.

To conclude this section we display the dependence of these groups on $\pi_{1}(M, *)$. Using either the left or right action of $\pi_{1}(M, *)$ on $\Lambda(M, *)$ we have $\Lambda(M, *) \simeq \Lambda(\widetilde{M}, \widetilde{*}) \times \pi_{1}(M, *)$, where $\widetilde{M}$ denotes the universal cover of $M$ and $\Lambda(\tilde{M}, \tilde{*})$ is naturally identified with the identity component of $\Lambda(M, *)$. Selecting the right action of $\pi_{1}(M, *)$, we can write $\Omega_{*}^{f r}(\Lambda(M, *))$ as a group ring:

$$
\Omega_{*}^{f r}(\Lambda(M, *)) \approx \Omega_{*}^{f r}(\Lambda(\tilde{M}, \tilde{*}))\left[\pi_{1}(M, *)\right]
$$

Under this isomorphism $a \in \Omega_{*}^{f r}(\Lambda(M, *))$ corresponds to a sum $\Sigma a_{\sigma} \sigma$, where $a_{\sigma}$ is the part of $a \cdot \sigma^{-1}$ supported by the identity component of $\Lambda(M, *)$. With this convention we have $\pi_{1}(M, *)$ acting on $\Omega_{*}^{f r}(\Lambda(\widetilde{M}, \widetilde{*}))\left[\pi_{1}(M, *)\right]$ by $\left(\Sigma a_{\sigma} \sigma\right) \tau=$ $\Sigma a_{\sigma} \sigma \tau$ and $\tau\left(\Sigma a_{\sigma} \sigma\right)=\Sigma\left(a_{\sigma}\right)^{\tau} \tau \sigma$, where ()$^{\tau}$ denotes $\tau \in \pi_{1}(M, *)$ acting on $\Omega_{*}^{f r}(\Lambda(\widetilde{M}, \tilde{*}))$ by conjugation. Also, $\overline{\Sigma a_{\sigma} \sigma}=\Sigma \omega_{1}(\sigma) \bar{a}_{\sigma} \sigma^{-1}$.

\subsection{Corollary.}

and

$$
\Omega_{0}^{f r}(\Lambda(M, *)) \approx \mathrm{Z}\left[\pi_{1}(M, *)\right]
$$

$$
\Omega_{1}^{f r}(\Lambda(M, *)) \approx\left(\mathrm{Z} / 2 \times \pi_{2}(M, *)\right)\left[\pi_{1}(M, *)\right] .
$$

For self-intersections, since $\omega: \Lambda(M, *) \rightarrow\{0$ or PL $\}$ is the product of its restriction $\widetilde{\omega}$ to $\Lambda(\widetilde{M}, \tilde{*})$ and $\omega_{1}: \pi_{1}(M, *) \rightarrow Z / 2$, we have

$$
\begin{aligned}
\Omega_{*}^{f r}\left(\Lambda(M, *) X_{2} W_{2} ;\right. & \left.\gamma^{k} \oplus e / \omega\right) \\
& \approx \Omega_{*}^{f r}\left(\Lambda(\tilde{M}, \tilde{*}) X_{2} W_{2} ; \gamma^{k+\omega_{1}} \oplus e / \tilde{\omega}\right)\left[\pi_{1}(M, *)\right] .
\end{aligned}
$$

Note that the group in which the coefficient of $\sigma$ lies depends on $\omega_{1}(\sigma)$, so this is not actually a group ring.

4. Isotopy and regular homotopy of subbundles. In this section we consider fiber regular homotopy (isotopy) of fiberwise immersions (embeddings) of differentiable or PL bundles.

To fix notation, we suppose $E_{M}, E_{Q}, E_{P}$ are smooth or PL locally trivial fiber bundles over a compact manifold $R^{k}$. The fibers are $M^{m}, P^{p}, Q^{q}$ respectively, and we assume $P, Q$ closed. $i_{P}: E_{P} \rightarrow E_{M}$ and $i_{Q}: E_{Q} \rightarrow E_{M}$ are bundle maps which are immersions (embeddings) in each fiber. 


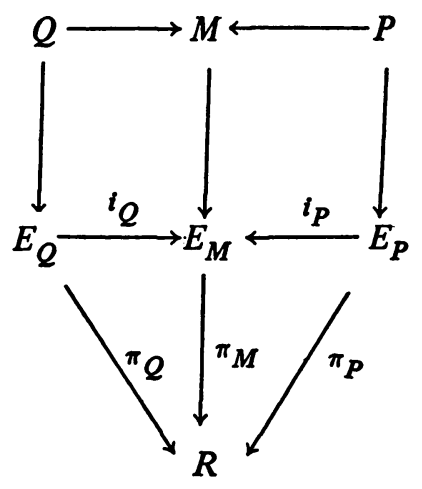

4.1. THEOREM. If $i_{Q}$ is fiber homotopic to a map transversal to $i_{P}$ with pullback $N$, by a homotopy fixed over $\partial R$, and $m>q+(p+k) / 2+1$, then there is a fiber regular homotopy (isotopy) fixed over $\partial R$ from $i_{Q}$ to an immersion (embedding) transversal to $i_{p}$ with pullback $N$.

Proof. The proof of 1.1 will carry over to the fibered case provided that the approximations of $H: E_{Q} \times I \rightarrow E_{M}$ which put it in general position can be made in a fiber preserving way.

Locally the bundles $E_{P}, E_{Q}$, and $E_{M}$ are trivial and $H$ takes the form $\left(\pi_{Q}, h\right)$ : $R \times Q \times I \rightarrow R \times(M, P)$. Since $\Sigma H \subset \Sigma h$, to make $\overline{\operatorname{sh}(\Sigma H)}$ disjoint from $H^{-1}(R \times P)$, for example, it suffices to make $\overline{\operatorname{sh}(\Sigma h)}$ disjoint from $h^{-1}(P)$. The condition $\operatorname{dim} \operatorname{sh}(\Sigma h)<\operatorname{codim} P$ is just $m>q+(p+k) / 2+1$ for immersions, $m>q+(p+k) / 2+3 / 2$ for embeddings. So approximating $h$ to make $\overline{\operatorname{sh}(\Sigma h)} \cap h^{-1}(P)=\varnothing$ leads to a fiberwise deformation of $\overline{\operatorname{sh}(\Sigma H)}$ off $H^{-1}(R \times P)$. Likewise, Case II of 1.1 translates to this setting.

It is then straightforward to fit together local fiberwise deformations of $H$ which put it in general position above pieces of $R$ over which the bundles are trivial, to make $H$ globally in general position with respect to $E_{P}$.

Note that if $P, Q$, and $M$ in 1.1 are replaced by $E_{P}, E_{Q}$, and $E_{M}$, the dimension restriction $m>q+p / 2+1$ becomes just $m>q+(p+k) / 2+$ 1. That is, the dimension hypothesis is really the same in the fibered case as in the unfibered case.

Next the bordism results of $\S 2$ are generalized to this setting. Since we now allow $E_{P}, E_{Q}$, and $E_{M}$ to have boundaries (over $\partial R$ ), we must modify our hypotheses accordingly. Recall the natural map $i_{P} \pi i_{Q} \rightarrow E\left(i_{P}, i_{Q}\right)$ covered by a bundle isomorphism

$$
v_{i_{P}} \pi_{Q} \rightarrow \nu\left(E_{P}, E_{M}\right) \oplus \nu\left(E_{Q}, E_{M}\right) \oplus v_{E_{M}} .
$$

Suppose given another manifold $N$ with such a map to $E\left(i_{P}, i_{Q}\right)$ and bundle 
isomorphism which agree on the common boundary $\partial N=\partial\left(i_{P} \bar{\pi} i_{Q}\right)$.

4.2. THEOREM. If $m>p+(q+k) / 2+1$ and $m>q+(p+k) / 2+1$ then $i_{Q}$ is fiber regularly homotopic (isotopic) holding boundary fixed to a fiber im-

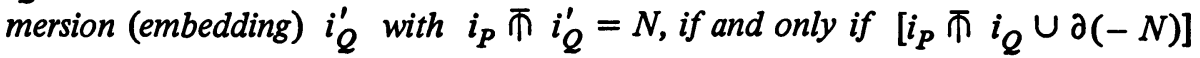
is zero in

$$
\Omega_{p+q+k-m}^{f r}\left(E\left(i_{P}, i_{Q}\right) ; \nu\left(E_{P}, E_{M}\right) \oplus \nu\left(E_{Q}, E_{M}\right) \oplus v_{E_{M}}\right) .
$$

Proof. As in 2.1, even a homotopy of $i_{Q}$ to such a map implies vanishing of the invariant. For the converse we construct a fiber homotopy and apply 4.1.

Let $W$ be a bordism from $i_{P} \bar{\pi} i_{Q}$ to $N$ trivial over $\partial\left(i_{P} \bar{\pi} i_{Q}\right)$. Then we have maps $j_{P}: W \rightarrow E_{P}$ and $j_{Q \times I}: W \rightarrow E_{Q} \times I$ with $j_{Q \times I}^{-1}\left(E_{Q} \times\{0\}\right)=$ $i_{P} \pi i_{Q}, j_{Q \times I}^{-1}\left(E_{Q} \times\{1\}\right)=N$, and we have a homotopy $h: i_{P} j_{P} \simeq i_{Q} \pi j_{Q \times I}$

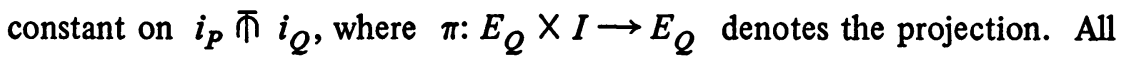
this data is trivial over $\partial\left(i_{P} \Phi i_{Q}\right)$.

We first change $j_{P}$ and $h$ so they are fiber preserving with respect to $\pi_{M}$. The composition $\pi_{M} h$ is a homotopy $\pi_{P} j_{P} \simeq \pi_{Q} \pi_{Q \times I}$, so in the diagram

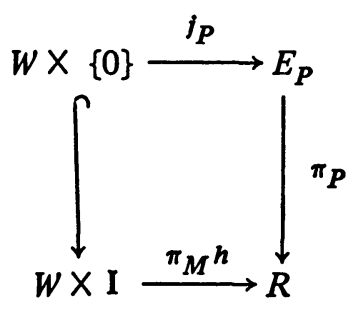

we can apply the homotopy lifting property for $\pi_{P}$ to get a homotopy of $j_{P}$ to $j_{P}^{\prime}$ with $\pi_{P} j_{P}^{\prime}=\pi_{Q} \pi_{Q \times I}$. Homotopy-composing this homotopy with the inverse homotopy of $h$, we obtain a homotopy from $i_{P} j_{P}^{\prime}$ to $i_{Q} \pi j_{Q \times I}$ whose projection is $\left(-\pi_{M} h\right) \cdot\left(\pi_{M} h\right)$. This homotopy is homotopic to the constant homotopy, so applying the lifting property for $\pi_{M}$ we get $h^{\prime}: i_{P} j_{P}^{\prime} \simeq i_{Q} \pi_{Q \times I}$ with $\pi_{M} h^{\prime}$ the constant homotopy. All this can be done preserving triviality on $i_{P} \pi i_{Q} \cup \partial\left(i_{P} \Phi i_{Q}\right) \times I$.

We can assume $j_{Q \times I}$ is an embedding (since $\operatorname{dim} W<1 / 2 \operatorname{dim}\left(E_{Q} \times I\right)$ ) with $\pi_{Q} \pi_{Q \times I}$ in general position. Then since $\operatorname{dim} W<1 / 2 \operatorname{dim} E_{P}$, we can approximate the new $j_{P}$ by an embedding in a fiber preserving way. Let $\tilde{j}_{Q \times I}$ : $F \rightarrow E_{Q} \times I$ be an embedding of a tubular neighborhood of $W$ in $E_{Q} \times I$, with $F_{0}=\tilde{j}_{Q \times I}^{-1}\left(E_{Q} \times\{0\}\right)$. Let $\tilde{j}_{M}: F \rightarrow E_{M}$ be projection on $W$ followed 
by $j_{P}$, but with the projection damped out near $F_{0}$ (partition of unity) so that on $F_{0} \widetilde{j}_{M}=i_{Q} \pi \widetilde{j}_{Q \times I}$, using the isomorphism

$$
F_{0} \approx \nu\left(i_{P} \pi i_{Q}, E_{Q}\right) \approx \nu\left(E_{P}, E_{M}\right) \mid i_{P} \pi i_{Q}
$$

The fiber preserving homotopy $h$ naturally extends to $\tilde{h}: \widetilde{j}_{M} \simeq i_{Q} \pi \widetilde{j}_{Q \times I}$, constant on $F_{0}$. Just as $h$ and $j_{P}$ were made fiber preserving, $\widetilde{h}$ and $\widetilde{j}_{M}$ can be made fiber preserving too.

The new $\widetilde{j}_{M}$ is transverse to $E_{P}$ only near $F_{0}$. To make $\tilde{j}_{M}$ transverse away from $F_{0}$ we perturb it near $W$ (but away from $F_{0}$ ) by adding $\left.\nu\left(E_{P}, E_{M}\right)\right|_{W}$ using the isomorphism $\left.\nu\left(E_{P}, E_{M}\right)\right|_{W} \approx \nu\left(W, E_{Q} \times I\right)=F$ given by the bundle data. Since the fibers of $\nu\left(E_{P}, E_{M}\right)$ can be taken to lie in the fibers of $\pi_{M}$, this perturbation $\tilde{j}_{M}$ of $\tilde{j}_{M}$ will still be fiber preserving with respect to $\pi_{M}$.

We can now define $H: E_{Q} \times I \rightarrow E_{M}$. Near the core of $F$ it is given by $\widetilde{j}_{M}^{\prime}$. Proceeding radially outward to the boundary of $F$ we first damp out the perturbation of $\widetilde{j}_{M}$ and then follow the homotopy $\tilde{h}: \widetilde{j}_{M} \simeq i_{Q} \pi \tilde{j}_{Q \times I}$, so that on the boundary of $F, H$ is $i_{Q} \pi \widetilde{j}_{Q \times I}$. Then $i_{Q} \pi$ can be used to define $H$ on $E_{Q} \times I-F$. The homotopy $H$ is fiber preserving, equals $i_{Q}$ on $E_{Q} \times\{0\}$, is constant on $\partial E_{Q} \times I$, and is transverse to $i_{P}$ on a smaller neighborhood $F^{\prime} \subset F$ of $W$.

The considerations of the proof of 4.1 can be applied to make $H$ transverse to $i_{P}$ everywhere preserving fibers and holding it fixed on $F^{\prime} \cup E_{Q} \times$ $\{0\}$. Thus $H^{-1}\left(E_{P}\right)=W \cup V$ with $V$ disjoint from $W$ and from $E_{Q} \times\{0\}$. Now imitate the proof of 1.1 to excise from $E_{Q} \times I$ a neighborhood of the (upward) shadow of $V$ disjoint from $W$. The restriction of $H$ to the remainder of $E_{Q} \times I$ is a fiber homotopy of $i_{Q}$ transverse to $i_{p}$ with pullback $W$, and 4.1 applies to produce a fiber regular homotopy or isotopy.

We remark again that the proof constructs a fiber regular homotopy (isotopy) of $E_{Q}$ intersecting $E_{P}$ exactly in $W$, which may also be preassigned.

The fibered analogue of the self-intersection case follows easily from this, as 2.3 does from 2.2. Again the dimension restrictions and obstructions are the same as the unfibered cases, so we will not give a statement.

As a sample application of this theory we use 4.2 to consider existence and uniqueness of nonzero sections of a bundle. Recall from $\S 3$ the definition of the bordism Euler class of a bundle over a manifold as the intersection of two sections of the bundle.

4.3. CoRollary. If $\xi$ is $a \mathrm{PL}$ or vector bundle of dimension $m$ over a closed $k$-manifold $R$ with $m>k / 2+1$, then $\xi$ has a nonvanishing section 
iff $\chi(\xi)=0$ in $\Omega_{k-m}^{f r}\left(R ; \xi \oplus \nu_{R}\right)$. If $\xi$ has a nonvanishing section and $m>k / 2+2$, then isotopy classes of nonzero sections (isomorphism classes of 1-dimensional subbundles) correspond bijectively with $\Omega_{k-m+1}^{f r}\left(R ; \xi \oplus \nu_{R}\right)$.

Proof. By 4.2, $\chi(\xi)=0$ implies there is a fiber isotopy of any section to one disjoint from the zero section. If $\xi$ has a section we map $\Omega_{k-m+1}^{f r}\left(R ; \xi \oplus \nu_{R}\right)$ to nonzero sections by finding a fiber isotopy taking the section to another one, so that the intersection of the isotopy with the 0 -section is a representative of the given bordism class. 4.2 shows that with the given dimension restriction this is well defined and a bijection.

Corollary 4.3 is just a new proof of the (well-known) fact:

4.4. COROLlary. The sequences

$$
B_{\mathrm{O}_{n-1}} \rightarrow B_{\mathrm{O}_{n}} \rightarrow T \gamma_{\mathrm{O}_{n}}, \quad B_{S_{n-1}} \rightarrow B_{S O_{n}} \rightarrow T \gamma_{S O_{n}}
$$

are homotopy fibrations up to dimension $2 n-2$.

Here $T \gamma_{X}$ denotes the Thom space of the universal $X$ bundle over $B_{X}$, $B_{X} \rightarrow T \gamma_{X}$ the natural inclusion. Homotopy fibration means, for example, the sequence induces a long exact sequence of homotopy groups up to the given dimension, or that it has the lifting property for nullhomotopic maps of $(2 n-3)$ complexes.

\section{REFERENCES}

1. A. Haefliger, Plongements différentiables dans le domaine stable, Comment. Math. Helv. 37 (1962/63), 155-176. MR 28 \#625.

2. A. Haefliger and V. Poenaru, La classification des immersions combinatoires, Inst. Hautes Étude Sci. Publ. Math. No. 23 (1964), 75-91. MR 30 \#2515.

3. M. W. Hirsch, Immersions of manifolds, Trans. Amer. Math. Soc. 93 (1959), 242-276. MR 22 \#9980.

4. J. F. P. Hudson, Piecewise linear topology, Benjamin, New York, 1969. MR $40 \# 2094$.

5. F. Laudenbach, Disjonction de sous-variétés et application au croisement des anses, Ann. Sci. École Norm. Sup. (4) 3 (1970), 385-408. MR 44 \#3332.

6. J. Stallings, Lectures on polyhedral topology, Tata Institute of Fundamental Research Lectures on Math., no. 43, Tata Institute of Fundamental Research, Bombay, 1967. MR 38 \#6605.

7. C. T. C. Wall, Surgery on compact manifolds, Academic Press, New York, 1970.

8. — Geometric connectivity. I, J. London Math. Soc. (2) 3 (1971), 597-604. MR 44 \#7569a.

9. R. Wells, Modifying intersections, Illinois J. Math. 11 (1967), 389-403. MR 36 \#896. 
10. A. Tineo, Un théorème de relèvement dans les spaces d'applications différentiables, Thèse de 3-ème cycle, Orsay, 1971.

11. J. P. Dax, Etude homotopique des espaces de plongements, Thèse, Orsay, 1971.

DEPARTMENT OF MATHEMATICS, PRINCETON UNIVERSITY, PRINCETON, NEW JERSEY 08540 (Current address of Allan Hatcher)

SCHOOL OF MATHEMATICS, INSTITUTE FOR ADVANCED STUDY, PRINCETON, NEW JERSEY 08540

Current address (Frank Quinn): Department of Mathematics, Yale University, New Haven, Connecticut 06520 